\title{
Identification and Characterization of the Roles of CircCASP9 in Gastric Cancer Based on a CircRNA- miRNA-mRNA Regulatory Network
}

Ziwei Wang ( $\sim$ ziweiwang1@sohu.com )

The First Affiliated Hospital of Chongqing Medical University https://orcid.org/0000-0003-3392-5483

Chuan Qin

The First Affiliated Hospital of Chongqing Medical University

Han Zhang

Chongqing University Three Gorges Hospital

Xiong Guo

The First Affiliated Hospital of Chongqing Medical University

Anqi Cheng

The First Affiliated Hospital of Chongqing Medical University

Primary research

Keywords: gastric cancer, circular RNA, circCASP9, regulatory network, tumorigenesis

Posted Date: February 23rd, 2021

DOI: https://doi.org/10.21203/rs.3.rs-239897/v1

License: (9) This work is licensed under a Creative Commons Attribution 4.0 International License.

Read Full License 
1 Identification and Characterization of the Roles of CircCASP9 in

2 Gastric Cancer Based on a CircRNA-miRNA-mRNA Regulatory

3 Network

4 Chuan Qin ${ }^{1,2 \dagger}$, Han Zhang ${ }^{3 \dagger}$, Huawen Liu ${ }^{3}$, Xiong Guo ${ }^{1}$, Anqi Cheng ${ }^{1}$, Ziwei Wang ${ }^{*}$

$5 *$ Correspondence:

6 ziweiwang1@ sohu.com

$7{ }^{1}$ Department of Gastrointestinal Surgery, The First Affiliated Hospital of Chongqing Medical

8 University, Chongqing, China

9 Full list of author information is available at the end of the article

10 These authors have contributed equally to this work and share first authorship. 


\section{Abstract}

24 Background: Circular RNAs (circRNAs) play crucial roles in gastric cancer (GC). This study aimed

25 to construct a new circRNA-miRNA-mRNA competing endogenous RNA (ceRNA) network for GC

26 and validate a specific circRNA in GC tissues and cell lines.

27 Methods: We obtained circRNA profiles from an in-house circRNA microarray and the Gene

28 Expression Omnibus (GEO) database, and microRNA (miRNA) profiles from StarBase and

29 CircBank. Messenger RNA profiles were downloaded from GEO. We then constructed a ceRNA

30 network using the extracted circRNA (circCASP9), miRNA (miR-589-5p) and 52 mRNAs. The

31 expression of circCASP9 was confirmed in GC tissues and cell lines, using quantitative reverse

32 transcription polymerase chain reaction. The function of circCASP9 was validated in GC cell lines,

33 using the cell counting kit-8, ethynyl deoxyuridine, transwell, and colon assays. The sponge function

34 of circCASP9 for miR-589-5p was verified using dual luciferase assays.

35 Results: A novel ceRNA network was established with one downregulated circRNA, one

36 upregulated miRNA, and 52 downregulated mRNAs. CircularCASP9 was downregulated in GC

37 tissues and cell lines and sponged miR-589-5p. Furthermore, circCASP9 inhibited GC proliferation,

38 migration, and invasion.

39 Conclusions: We identified the expression and anticancer effects of the core circRNA circCASP9 in

40 a novel circRNA-miRNA-mRNA ceRNA regulatory network of GC. This present study may provide

41 a novel therapeutic target and insight understanding of the mechanism of gastric cancer.

42 Keywords: gastric cancer, circular RNA, circCASP9, regulatory network, tumorigenesis

\section{Background}


44 Gastric cancer (GC) ranks fifth among the most prevalent cancers and accounts for $\sim 33 \%$ of cancer-

45 related mortality globally [1]. Most patients with GC have an advanced stage of the disease at the

46 time of diagnosis [2]. Despite progress in GC treatment, the 5-year survival rate remains at 20-25\%

47 [3]. Thus, exploring the mechanism of tumorigenesis and tumor development is important for the 48 early diagnosis and therapy of GC. Circular RNAs (circRNAs) are a recently discovered category of

49 endogenous noncoding RNAs, formed by the covalent bonding of 5' and 3 ' ends and generated by

50 reverse splicing introns or exons $[4,5]$. Circular RNAs are primarily located in the cytoplasm and are

51 competing endogenous RNAs (ceRNAs). Circular RNAs can competitively sponge microRNAs

52 (miRNAs) to regulate mRNA expression, thereby affecting the genesis and progression of tumors

53 [6-8]. For instance, circREPS2 inhibits GC progression by combining with miR-558 [9]. The

54 circRNA MCTP2 suppresses cisplatin resistance in GC by absorbing miR-99a-5p [10]. Circular

55 LMO7 promotes GC progression by acting on miRNA-30a-3p [11]. Other circRNAs associated with

56 GC should be identified to further increase the understanding of the potential molecular mechanisms 57 of GC.

Here, we generated data from a new microarray and the online databases, to identify a candidate

59 circRNA associated with GC. Based on predicted interactions among circRNAs, miRNAs, and

60 mRNAs, we established a novel ceRNA network. Moreover, we explored the expression and function

61 of circCASP9 and verified the sponge function of circCASP9 to miR-589-5p in the network.

\section{Methods}

\section{Data sources}

64 Our circRNA microarray was manufactured by CapitalBio Technology (Beijing, China). Two

65 additional circRNA expression datasets, GSE89143 and GSE83521, were collected from the Gene

66 Expression Omnibus (GEO) database (https://www.ncbi.nlm.nih.gov/geo/) [12]. Circular RNAs that 
67 could not be transformed to standard names in circBase were excluded [13]. MicroRNA profiles were 68 obtained from the StarBase (http://starbase.sysu.edu.cn/), circBank (http://www.circbank.cn/), and

69 CircInteractome (https://circinteractome.nia.nih.gov/) databases [14-16]. The mRNAs were acquired

70 from the StarBase, MiRDB (http://www.mirdb.org/), and TargetScan databases

71 (http://www.targetscan.org/) [17, 18], and mRNA expression profiles were downloaded from the

72 GEO dataset GSE33651. The network diagrams for circRNAs were downloaded from the Cancer-

73 Specific circRNA Database (CSCD) (http://gb.whu.edu.cn/) [19].

\section{CircRNA microarray analysis}

75 Our circRNA microarray of five pairs of fresh GC and paracancerous tissues was manufactured by

76 CapitalBio Technology (Beijing, China). Total RNA was extracted with TRIzol reagent (Invitrogen,

77 Carlsbad, CA, USA). CircRNAs were enriched after the total RNA was digested with RNase R

78 (Epicentre; Illumina, Inc., San Diego, CA, USA). After amplification, the enriched circRNAs were

79 transcribed into fluorescent cDNA. Then, the cdna was hybridized using Hybridization Oven

80 (Agilent Techologies, G2545A). Next, the washed microarray was scanned by Agilent Microarray

81 Scanner (Agilent Techologies, G2565A). Finally, the microarray data was collected by Agilent

82 Feature Extraction (v10.7) and were normalized by Agilent GeneSpring (Agilent Techologies, CA, 83 USA).

\section{Clinical specimens}

85 We obtained 40 pairs of GC and paracancerous tissues from the First Affiliated Hospital of 86 Chongqing Medical University, between 2017 and 2020. None of the patients had received 87 preoperative chemoradiotherapy. The tissue samples were immediately stored at $-80{ }^{\circ} \mathrm{C}$ after 88 surgical resection and validated by two pathologists.

\section{Cell culture}


The human GC cell lines AGS, MGC-803, and MNK-45, as well as a human gastric epithelial cell

91 line (GES-1) (Type Culture Collection of the Chinese Academy of Sciences, Beijing, China) were

92 incubated in RPMI 1640 (Biological Industry Ltd., Haemek, Israel), containing 10\% fetal bovine

93 serum (FBS) (Biological Industry Inc.), at $37^{\circ} \mathrm{C}$ under a $5 \% \mathrm{CO}_{2}$ atmosphere.

94 Construction of a circRNA-miRNA-mRNA network

95 We identified target circRNAs (TCs) using our circRNA microarray and the GSE89143 and

96 GSE83521 datasets. The upregulated and downregulated circRNAs identified from overlap among

97 the three microarrays were confirmed as TCs, using the "limma" R package (|log2[fold-change]|

$98[|\log \mathrm{FC}|]>2$ and adj.p < 0.05). The miRNAs sponged by circRNAs were predicted using StarBase

99 and circBank. Differentially expressed miRNAs were extracted from StarBase and were regarded as

100 target miRNAs (TMIs) (FC $>2, \mathrm{p}<0.05$ ). Differentially expressed mRNAs (DEMs) were identified

101 in the GSE33651 dataset using the "limma" $R$ package $(|\log F C|>1$ and adj.p $<0.05)$. Interactions

102 between MiRNA-mRNA were collected using StarBase, MiRDB, and TargetScan. The intersection

103 of predicted mRNAs from the three databases was obtained to identify target mRNAs (TMs). The

104 circRNA-miRNA-mRNA network was generated using Cytoscape version 3.8.0

105 (https://cytoscape.org/) [20].

106 Functional enrichment analysis of TMs

107 Overrepresented gene annotation terms in biological process (BP), cellular component (CC), and

108 molecular function (MF) categories were determined with gene ontology (GO) analysis [21].

109 Pathway enrichment was evaluated using the Kyoto Encyclopedia of Genes and Genomes (KEGG),

110 with thresholds for significance of $p<0.05$ and $q<0.05$ [22]. The results were visualized using the

111 clusterProfiler package in $\mathrm{R}$ [23].

\section{RNA extraction and qRT-PCR}


113 Total RNA isolated using TRIzol (Takara, Japan) was reverse transcribed using the PrimeScript RT

114 Reagent Kit (RR037A;Takara Bio Inc., Kusatsu, Japan) and analyzed with qRT-PCR using TB Green

115 Premix Ex TaqII (RR820A; Takara Bio Inc.). Divergent primers for circCASP9 were manufactured

116 by GeneSeed (Guangzhou, China), and other primers were purchased from Sangon Biotech

117 (Shanghai, China). Relative expression was determined using the $2^{-\Delta \Delta \mathrm{ct}}$ method.

\section{Oligonucleotide transfection}

119 CircCASP9-specific small interfering RNA (si-circCASP9) and normal control small interfering

120 RNA (si-NC) were designed by GenePharma (Shanghai, China). RiboFECT ${ }^{\text {TM }}$ CP (RiboBio,

121 Guangzhou, China) was used to transfect siRNA, as described by the manufacturer. The silencing

122 efficiency of si-circCASP9 was verified with qRT-PCR. Table 1 shows the primer sequences.

\section{Fluorescence in situ hybridization (FISH) assay}

124 We detected Cy3-labeled circCASP9 probes (GenePharma) in GC cells using FISH kits (RiboBio).

125 Probes specific to circCASP9 were mixed and incubated overnight, then nuclei were stained with

126 4',6-diamidino-2-phenyl indole (DAPI). Images were acquired using an LSM800 laser scanning

127 confocal microscope (Carl Zeiss Microscopy GmbH, Jena, Germany). Table 1 shows the sequences 128 of Cy3-labeled circCASP9 probes.

\section{Cell proliferation and colony-formation assays}

130 Transfected GC cells $\left(2 \times 10^{3} /\right.$ well $)$ were cultured in 96 -well plates, then incubated with $10 \mu \mathrm{L}$ of the

131 CCK-8 reagent (MedChemExpress, Monmouth Junction, NJ, USA) for $2 \mathrm{~h}$. Absorbance at $450 \mathrm{~nm}$

132 was detected at $0,24,48$, and $72 \mathrm{~h}$ thereafter.

133 Transfected GC cells $(6 \times 102 /$ well $)$ were cultured in 6-well plates for $8 \mathrm{~d}$, fixed in

134 paraformaldehyde (Servicebio, Wuhan, China), and stained with crystal violet (Solarbio Co., Ltd., 
135 Beijing, China). Thereafter, colony formation was assayed using a CanoScan 9000F Mark II scanner

136 (Canon Inc., Tokyo, Japan).

\section{Ethynyl deoxyuridine assays}

138 Transfected GC cells $\left(2 \times 10^{3} /\right.$ well $)$ were cultured in 96-well plates until they reached the logarithmic

139 growth phase. Cell viability was evaluated using Ethynyl Deoxyuridine (EdU) Labeling/Detection

140 Kits (RiboBio), as described by the manufacturer. Images were captured using an LSM800 laser

141 scanning confocal microscope.

\section{Migration and invasion assays}

143 Corning® Transwell@ cell culture chambers (Corning Inc., Corning, NY, USA) were embedded in

$144100 \mu \mathrm{L}$ of $1 \mathrm{mg} / \mathrm{mL}$ Matrigel matrix (BD Biosciences, San Jose, CA, USA). Thereafter, transfected

145 GC cells $\left(3 \times 10^{4} /\right.$ well $)$ in $300 \mu \mathrm{L}$ of serum-free medium, were seeded in the upper chambers and

146 incubated for $24 \mathrm{~h}$, with $700 \mu \mathrm{L}$ of medium containing $10 \%$ FBS in the lower chambers. Thereafter,

147 the cells were fixed in paraformaldehyde, stained with crystal violet, then visualized using an

148 inverted microscope (Leica).

\section{Dual-Luciferase reporter assay}

150 The circCASP9-wild-type (WT) plasmid with the full-length sequence and the circCASP9-mutant

151 (MUT) plasmid (pmirGLO-Dual-luciferase) without miR-589-5p binding sites were obtained from

152 Gene Create (Wuhan, China; Fig. 8e). MicroRNA-589-5p mimics and miR-NC were purchased from

153 Sangon Biotech (Shanghai, China). The plasmids and miR-589-5p mimics, or miR-NC were co-

154 transfected into MGC-803 cells, using Lipofectamine 2000 (Invitrogen, Carlsbad, CA, USA), and

155 incubated for $48 \mathrm{~h}$. The results were assessed using Dual-Luciferase Assay Kits (Beyotime).

\section{Statistical analysis}


157 Data were analyzed using R version 3.6.1 (http://www.R-project.org) and GraphPad Prism version 7

158 (https://www.graphpad.com/) [24]. Groups were compared using two-tailed Student t-tests or

159 analyses of variance (ANOVA). A heatmap and volcano plot were generated using the "pheatmap"

160 package in R to visually display differentially expressed circRNAs (DECs) and DEMs. Venn

161 diagrams were constructed using the Venn package in R to visualize overlapping circRNAs and

162 mRNAs. Values with $\mathrm{p}<0.05$ were considered as statistically significant.

\section{Results}

164 Identification of TCs, TMIs, and TMs

165 Our circRNA microarray contained 170,417 circRNAs. We acquired 484 DECs from our microarray

166 (filtered by $|\log \mathrm{FC}|>2$ and adj.p < 0.05), comprising 349 downregulated and 135 upregulated

167 circRNAs (Fig. 1a and b). Two overlapping circRNAs were identified from our microarray and the

168 GSE89143 and GSE83521 datasets (Fig. 1c and d). The circRNAs hsa_circ_0010039 (circCASP9)

169 and hsa_circ_0006649 (circDLG5) were downregulated (Table 2 and Fig. 1e-h).

170 Four binding miRNAs (miR-589-5p, miR-574-5p, miR-510-5p, and miR-668-3p) downstream of

171 the DECs were predicted from the overlapping miRNAs in StarBase, circBank, and CircInteractome

172 (Table 3, Fig. 2a-f). Among the overlapping miRNAs identified using expression data from the

173 StarBase database, miR-574-5p, miR-510-5p, and miR-668-3p were not significantly upregulated,

174 owing to a negative correlation between expression levels of the circRNA and corresponding

175 miRNA. We therefore excluded miR-574-5p, miR-510-5p, and miR-668-3p and the upstream

176 circDLG5. The expression of miR-589-5p was significantly upregulated in GC tissues according to

177 StarBase. Thus, miR-589-5p was regarded as a TMI, and circCASP9 as a TC.

178 We obtained 3,069 downregulated and 2,916 upregulated DEMs from the microarray and the

179 GSE33651 $(\log F C>1$ and FDR < 0.05) dataset (Fig. 3a, b). Finally, 52 mRNAs bound to miR-589- 
$1805 \mathrm{p}$ were obtained from the intersection of DEMs in StarBase, miRDB, and TargetScan (Fig. 3c, d).

181 The 52 overlapping mRNAs were identified as TMs.

\section{Construction of a ceRNA network}

183 We constructed a circRNA-miRNA-mRNA ceRNA network comprising one downregulated

184 circRNA, one upregulated miRNA, and 52 downregulated mRNAs. The circCASP9 was the ceRNA

185 of miRNA-589-5p, and miRNA-589-5p targeted the 52 mRNAs. The members of the ceRNA

186 network formed 52 regulated axes (Fig. 4).

\section{Functional enrichment analysis of TMs}

188 The 52 TMs were most significantly enriched in GO-BP terms associated with the cellular response

189 to osmotic stress, negative regulation of muscle adaptation, and cellular hyperosmotic response; GO-

190 CC terms were related to integral components of the presynaptic active zone membrane, intrinsic

191 components of the presynaptic active zone membrane, and the presynapse; GO-MF terms were

192 related to calmodulin-dependent protein kinase activity, sodium channel regulator activity, and

193 histone acetyltransferase activity $(\mathrm{p}<0.01$; Fig. 5a-c). The three most significant KEGG pathways

194 involving these genes were the phospholipase D signaling, adrenergic signaling in cardiomyocytes,

195 and Wnt signaling pathways ( $<<0.01$; Fig. 5d).

\section{Validation of CircCASP9 expression}

197 Based on the bioinformatic findings, we extracted circCASP9 (located at chr1: 15844604-15844890)

198 as the TM for further investigation. Sanger sequencing results showed that the circular structure of

199 circCASP9 was formed by reverse stitching (Fig. 6a). Compared with paired adjacent normal tissues,

200 we discovered less circCASP9 expression in 40 GC tissues (Fig. 6b) and in the MGC-803 and AGS

201 GC cell lines than in the human gastric epithelial cell line GES-1 (Fig. 6c). The findings of FISH

202 assays revealed that circCASP9 was located in the cytoplasm and nuclei of AGS and MGC-803 cells 
203 (Fig. 6d). Transfection with si-circCASP9 in MGC-803 and AGS cells resulted in significant

204 silencing efficacy (Fig. 6e, f).

205 CircCASP9 suppressed GC cell proliferation, colony formation, and DNA synthesis

206 The CCK-8 results revealed more multiplication capacity in the AGS and MGC-803 cells transfected

207 with si-circCASP9 than in the negative control si-NC group (Fig. 7a and b). Similarly, EdU assays

208 indicated that circCASP9 silencing increased DNA synthesis (Fig. 7c-e). Transfection with si-

209 circCASP9 increased colony-forming capacity (Fig. 7f and g). These results showed that circCASP9

210 inhibits GC cell activity and proliferation.

211 CircCASP9 inhibited the migration and invasion of GC cells

212 Silencing circCASP9 improved the migration and invasion ability of AGS and MGC-803 cells

213 compared with that of the negative control si-NC. Therefore, circCASP9 inhibited GC cell migration 214 and invasion (Fig. 8a-d).

215 CircCASP9 acted as an miR-589-5p sponge

216 Luciferase activity of circCASP9-WT was obviously decreased by miR-589-5p mimics in MGC-803

217 cells compared with the circCASP9-MUT or miR-589-5p NC groups (Fig. 8e and f).

\section{Discussion}

219 Circular RNAs were originally regarded as non-coding RNAs produced due to splicing errors.

220 However, circRNAs are in fact a large class of endogenous, conserved non-coding RNA produced by

221 back-splicing $[25,26]$. The covalent cyclic structure of circRNA is more stable than that of linear

222 RNA [27]. Owing to the discovery of a wide range of biological and pathological functions,

223 circRNAs have become a major focus of recent research [28]. 
Circular RNAs have crucial regulatory effects on various biological activities, particularly cancer

225

226

227

228

229

230

231

232

233

234

235

236

237

238

239

240

241

242

243

244

occurrence and progression [29-31]. They sponge miRNAs to modulate gene expression, which is the main pathway through which tumor progression is regulated [32]. For example, circCCDC9 can suppress the development of GC via the miR-6792-3p/CAV1 axis [33], circLMTK2 can lead to GC growth and metastasis by regulating the miR-150-5p/c-Myc axis [34], and circLARP4 can bind with miR-424-5p to inhibit GC development [35]. However, the mechanisms through which circRNAs contribute to GC remain largely unknown.

We created a ceRNA network comprising one circRNA, one miRNA, and 52 mRNAs, to identify core circRNAs in GC and their potential molecular targets. Circular CASP9 was generated by back splicing CASP9 (caspase 9) exon 10. The initiator caspase CASP9 is involved in intrinsic apoptosis as well as macroautophagy/autophagy regulation [36]. To our knowledge, this is the first study to identify a close relationship between circCASP9 and GC.

We confirmed that circCASP9 is downregulated in GC tissue samples and cell lines. This was consistent with the results from our circRNA microarray. The results of a series of in vitro functional assays to determine the underlying molecular mechanisms of circCASP9, showed that it suppressed GC cell proliferation, colony formation, DNA synthesis, migration, and invasion. Furthermore, we focused on miR-589-5p as the target miRNA of circCASP9. The consequences indicated that circCASP9 might be associated with GC tumorigenesis and development, by sponging miR-589-5p. Circular RNAs typically inhibit mRNA expression by binding directly to target miRNAs in the cytoplasm. The findings of FISH assays showed that circCASP9 was located in the cytoplasm and nuclei.

We found using the ceRNA network that miR-589-5p, which promotes GC metastasis via regulating the PIK3K/AKT pathway [37], binds to circCASP9. This ceRNA network could provide 
insights into circRNA-miRNA interactions and valuable information for future studies of miRNAs in 248 GC.

249 We identified 52 downstream genes that were predicted to bind to mir-589-5p. Gene ontology 250 analysis revealed that these genes were significantly related to the following GO terms: cellular

251 response to osmotic stress, integral component of presynaptic active zone membrane, and

252 calmodulin-dependent protein kinase activity. The results of KEGG analysis revealed a relationship

253 with 12 signaling pathways, including those associated with tumor-related processes. The

254 phospholipase D signaling pathway affects tumorigenesis [38], the activated Wnt signaling pathway

255 promotes GC cell proliferation, metastasis, and invasion [39, 40], the activated calcium signaling

256 pathway can inhibit non-small lung cancer cell proliferation and promote cell apoptosis [41], and

257 choline metabolism is associated with cancer tumorigenesis and might be a therapeutic target [42].

258 We constructed a novel ceRNA network for GC and identified circCASP9 as a core circRNA.

259 We confirmed circCASP9 expression in GC tissues and cell lines, and that it regulates the growth,

260 migration, and invasion of GC cells. Moreover, circCASP9 acted as a sponge of miR-589-5p in GC

261 cells. However, this study has several limitations. Firstly, the association between circCASP9

262 expression and prognosis could not be clinically validated because of insufficient clinical samples

263 with adequate follow-up. Furthermore, the mechanism(s) underlying the effects of circCASP9 in GC

264 should be investigated in vivo and in vitro.

\section{Conclusions}

266 Our findings indicate that circCASP9, a core circRNA identified using a circRNA-miRNA-mRNA

267 network, plays significant roles in GC cell growth and invasion. Therefore, circCASP9 might be a

268 useful target for further investigation into the occurrence and development of GC and for the design

269 of individualized therapeutic strategies. 
270 Abbreviations

271 BP Biological process

272 CC Cellular component

273 CCK-8 counting kit-8

274 ceRNA Competing endogenous RNA

275 circRNAs circular RNA

276 DAPI 4',6-diamidino-2-phenyl indole

277 DECs Differentially expressed circRNAs

278 DEMs Differentially expressed mRNAs

279 EdU Ethynyl Deoxyuridine

280 FBS Fetal bovine serum

281 FISH Fluorescence in situ hybridization

282 GC Gastric cancer

283 GEO Gene Expression Omnibus

284 GO Gene ontology

285 KEGG Kyoto Encyclopedia of Genes and Genomes

286 MF Molecular function

287 miRNAs microRNAs

288 MUT mutant

289 qRT-PCR quantitative reverse transcription polymerase chain reaction

290 si-circCASP9 small interfering RNA

291 si-NC normal control small interfering RNA

292 TCs Target circRNAs

293 TMIs Target miRNAs

294 TMs Target mRNAs

295 WT wild-type 
Declarations

\section{Acknowledgements}

298 Not applicable.

299 Authors' Contributions

$300 \mathrm{CQ}$ and $\mathrm{HZ}$ contributed to research concept, design, experiments, and manuscript writing. XG and

$301 \mathrm{HL}$ analyzed the data and edited the figures and tables. AC collected tissues. ZW supervised the

302 study. All authors read and approved the final manuscript.

\section{$303 \quad$ Funding}

304 This study was supported by the National Natural Science Foundation of China (NSFC; no:

$30581974385)$.

\section{Availability of data and materials}

307 The GSE89143 and GSE83521 datasets can be found at Gene Expression Omnibus

308 (https://www.ncbi.nlm.nih.gov/geo/). The circRNA sequence was acquired from circBase

309 (http://www.circbase.org/), and the network diagrams for circRNAs are in the Cancer-Specific

310 CircRNA database (http://gb.whu.edu.cn/). The miRNA profiles are in StarBase

311 [http://starbase.sysu.edu.cn/]. The mRNA datasets are in StarBase (http://starbase.sysu.edu.cn/),

312 MiRDB (http://www.mirdb.org/), and TargetScan (http://www.targetscan.org/). All data generated or

313 analyzed during the current study are available from the corresponding author on reasonable request.

\section{Ethics approval and consent to participate}

315 The Ethics Committee of Chongqing Medical University approved the present study. All participants 316 provided written informed consent before the study started.

317 Consent for publication 
318 Not applicable.

319 Competing interests

320 The authors declare that they have no competing interests.

321 Authors' information

322 Chuan Qin and Han Zhang have contributed equally to this work and share first authorship.

323 Affiliations

324 Department of Gastrointestinal Surgery, The First Affiliated Hospital of Chongqing Medical

325 University, Chongqing, China.

326 Chuan Qin, Xiong Guo, Anqi Cheng \& Ziwei Wang

327 Department of Intestinal Surgery, Chongqing University Three Gorges Hospital, Wanzhou,

328 Chongqing, China.

329 Chuan Qin

330 Department of Oncology, Chongqing University Three Gorges Hospital, Wanzhou, Chongqing,

$331 \quad$ China

332 Han Zhang and Huawen Liu

\section{References}

1. Smyth E, Nilsson M, Grabsch H, van Grieken N, Lordick F: Gastric cancer. Lancet (London, England) 2020, 396(10251):635-648.

2. Hunt R, Camilleri M, Crowe S, El-Omar E, Fox J, Kuipers E, Malfertheiner P, McColl K, Pritchard D, Rugge M et al: The stomach in health and disease. Gut 2015, 64(10):16501668.

3. Niccolai E, Taddei A, Prisco D, Amedei A: Gastric cancer and the epoch of immunotherapy approaches. World journal of gastroenterology 2015, 21(19):57785793.

4. Wu W, Zhen T, Yu J, Yang Q: Circular RNAs as New Regulators in Gastric Cancer: Diagnosis and Cancer Therapy. Frontiers in oncology 2020, 10:1526.

5. Chen L: The biogenesis and emerging roles of circular RNAs. Nature reviews Molecular cell biology 2016, 17(4):205-211.

6. Li F, Yang Q, He A, Yang B: Circular RNAs in cancer: limitations in functional studies 
and diagnostic potential. Seminars in cancer biology 2020.

348 7. Han B, Chao J, Yao H: Circular RNA and its mechanisms in disease: From the bench to the clinic. Pharmacology \& therapeutics 2018, 187:31-44.

8. Guo J, Agarwal V, Guo H, Bartel D: Expanded identification and characterization of mammalian circular RNAs. Genome biology 2014, 15(7):409.

9. Guo X, Dai X, Liu J, Cheng A, Qin C, Wang Z: Circular RNA circREPS2 Acts as a Sponge of miR-558 to Suppress Gastric Cancer Progression by Regulating RUNX3/ $\beta$-catenin Signaling. Molecular therapy Nucleic acids 2020, 21:577-591.

10. Dai X, Guo X, Liu J, Cheng A, Peng X, Zha L, Wang Z: Circular RNA circGRAMD1B inhibits gastric cancer progression by sponging miR-130a-3p and regulating PTEN and p21 expression. Aging 2019, 11(21):9689-9708.

11. Cao J, Zhang X, Xu P, Wang H, Wang S, Zhang L, Li Z, Xie L, Sun G, Xia Y et al: Circular RNA circLMO7 acts as a microRNA-30a-3p sponge to promote gastric cancer progression via the WNT2/ $\beta$-catenin pathway. Journal of experimental \& clinical cancer research : $C R$ 2021, 40(1):6.

12. Barrett T, Troup D, Wilhite S, Ledoux P, Evangelista C, Kim I, Tomashevsky M, Marshall K, Phillippy K, Sherman $\mathrm{P}$ et al: NCBI GEO: archive for functional genomics data sets--10 years on. Nucleic acids research 2011, 39:D1005-1010.

13. Glažar P, Papavasileiou P, Rajewsky N: circBase: a database for circular RNAs. RNA (New York, NY) 2014, 20(11):1666-1670.

14. Li J, Liu S, Zhou H, Qu L, Yang J: starBase v2.0: decoding miRNA-ceRNA, miRNA-ncRNA and protein-RNA interaction networks from large-scale CLIP-Seq data. Nucleic acids research 2014, 42:D92-97.

15. Liu M, Wang Q, Shen J, Yang B, Ding X: Circbank: a comprehensive database for circRNA with standard nomenclature. RNA biology 2019, 16(7):899-905.

16. Dudekula D, Panda A, Grammatikakis I, De S, Abdelmohsen K, Gorospe M: CircInteractome: A web tool for exploring circular RNAs and their interacting proteins and microRNAs. RNA biology 2016, 13(1):34-42.

17. Kern F, Krammes L, Danz K, Diener C, Kehl T, Küchler O, Fehlmann T, Kahraman M, Rheinheimer S, Aparicio-Puerta $\mathrm{E}$ et al: Validation of human microRNA target pathways enables evaluation of target prediction tools. Nucleic acids research 2020.

18. Chen Y, Wang X: miRDB: an online database for prediction of functional microRNA targets. Nucleic acids research 2020, 48:D127-D131.

19. Xia S, Feng J, Chen K, Ma Y, Gong J, Cai F, Jin Y, Gao Y, Xia L, Chang H et al: CSCD: a database for cancer-specific circular RNAs. Nucleic acids research 2018, 46:D925-D929.

20. Reimand J, Isserlin R, Voisin V, Kucera M, Tannus-Lopes C, Rostamianfar A, Wadi L, Meyer $\mathrm{M}$, Wong J, $\mathrm{Xu} \mathrm{C}$ et al: Pathway enrichment analysis and visualization of omics data using g:Profiler, GSEA, Cytoscape and EnrichmentMap. Nature protocols 2019, 14(2):482-517.

21. Ashburner M, Ball C, Blake J, Botstein D, Butler H, Cherry J, Davis A, Dolinski K, Dwight S, Eppig J et al: Gene ontology: tool for the unification of biology. The Gene Ontology Consortium. Nature genetics 2000, 25(1):25-29.

22. Kanehisa M, Goto S: KEGG: kyoto encyclopedia of genes and genomes. Nucleic acids research 2000, 28(1):27-30.

23. Yu G, Wang L, Han Y, He Q: clusterProfiler: an R package for comparing biological themes among gene clusters. Omics : a journal of integrative biology 2012, 16(5):284287.

24. Incerti D, Thom H, Baio G, Jansen J: R You Still Using Excel? The Advantages of Modern 
Software Tools for Health Technology Assessment. Value in health : the journal of the International Society for Pharmacoeconomics and Outcomes Research 2019, 22(5):575579.

25. Chen L: The expanding regulatory mechanisms and cellular functions of circular RNAs. Nature reviews Molecular cell biology 2020, 21(8):475-490.

26. Kristensen L, Andersen M, Stagsted L, Ebbesen K, Hansen T, Kjems J: The biogenesis, biology and characterization of circular RNAs. Nature reviews Genetics 2019, 20(11):675-691.

27. Jeck W, Sharpless N: Detecting and characterizing circular RNAs. Nature biotechnology 2014, 32(5):453-461.

28. Patop I, Wüst S, Kadener S: Past, present, and future of circRNAs. The EMBO journal 2019, 38(16):e100836.

29. Peng Y, Wang H: Cir-ITCH inhibits gastric cancer migration, invasion and proliferation by regulating the $\mathbf{W n t} / \boldsymbol{\beta}$-catenin pathway. Scientific reports 2020, 10(1):17443.

30. Cheng D, Wang J, Dong Z, Li X: Cancer-related circular RNA: diverse biological functions. Cancer cell international 2021, 21(1):11.

31. Li W, Liu J, Chen M, Xu J, Zhu D: Circular RNA in cancer development and immune regulation. Journal of cellular and molecular medicine 2020.

32. Hansen T, Jensen T, Clausen B, Bramsen J, Finsen B, Damgaard C, Kjems J: Natural RNA circles function as efficient microRNA sponges. Nature 2013, 495(7441):384-388.

33. Luo Z, Rong Z, Zhang J, Zhu Z, Yu Z, Li T, Fu Z, Qiu Z, Huang C: Circular RNA circCCDC9 acts as a miR-6792-3p sponge to suppress the progression of gastric cancer through regulating CAV1 expression. Molecular cancer 2020, 19(1):86.

34. Wang $S$, Tang $D$, Wang $W$, Yang $Y, W u X$, Wang $L$, Wang D: circLMTK2 acts as a sponge of miR-150-5p and promotes proliferation and metastasis in gastric cancer. Molecular cancer 2019, 18(1):162.

35. Zhang J, Liu H, Hou L, Wang G, Zhang R, Huang Y, Chen X, Zhu J: Circular RNA_LARP4 inhibits cell proliferation and invasion of gastric cancer by sponging miR-424-5p and regulating LATS1 expression. Molecular cancer 2017, 16(1):151.

36. An H, Chung K, Park H, Hong J, Gim J, Choi H, Lee Y, Choi J, Mun J, Yu S: CASP9 (caspase 9) is essential for autophagosome maturation through regulation of mitochondrial homeostasis. Autophagy 2020, 16(9):1598-1617.

37. Zhang F, Li K, Pan M, Li W, Wu J, Li M, Zhao L, Wang H: miR-589 promotes gastric cancer aggressiveness by a LIFR-PI3K/AKT-c-Jun regulatory feedback loop. Journal of experimental \& clinical cancer research : CR 2018, 37(1):152.

38. Kang D, Min dS: Positive feedback regulation between phospholipase $D$ and Wnt signaling promotes Wnt-driven anchorage-independent growth of colorectal cancer cells. PloS one 2010, 5(8):e12109.

39. Fan D, Ren B, Yang X, Liu J, Zhang Z: Upregulation of miR-501-5p activates the wnt/ $\boldsymbol{\beta}$ catenin signaling pathway and enhances stem cell-like phenotype in gastric cancer. Journal of experimental \& clinical cancer research : CR 2016, 35(1):177.

40. Gao J, Zhao C, Liu Q, Hou X, Li S, Xing X, Yang C, Luo Y: Cyclin G2 suppresses Wnt/ $\beta$ catenin signaling and inhibits gastric cancer cell growth and migration through Dapper1. Journal of experimental \& clinical cancer research : CR 2018, 37(1):317.

41. Wang W, Mao L, Lai H, Wang Y, Jiang Z, Li W, Huang J, Xie Y, Xu C, Liu P et al: Dolutegravir derivative inhibits proliferation and induces apoptosis of non-small cell lung cancer cells via calcium signaling pathway. Pharmacological research 2020, 161:105129. 
443 42. Glunde K, Penet M, Jiang L, Jacobs M, Bhujwalla Z: Choline metabolism-based molecular 444 diagnosis of cancer: an update. Expert review of molecular diagnostics 2015, 15(6):735747. 


\section{Figures}

a

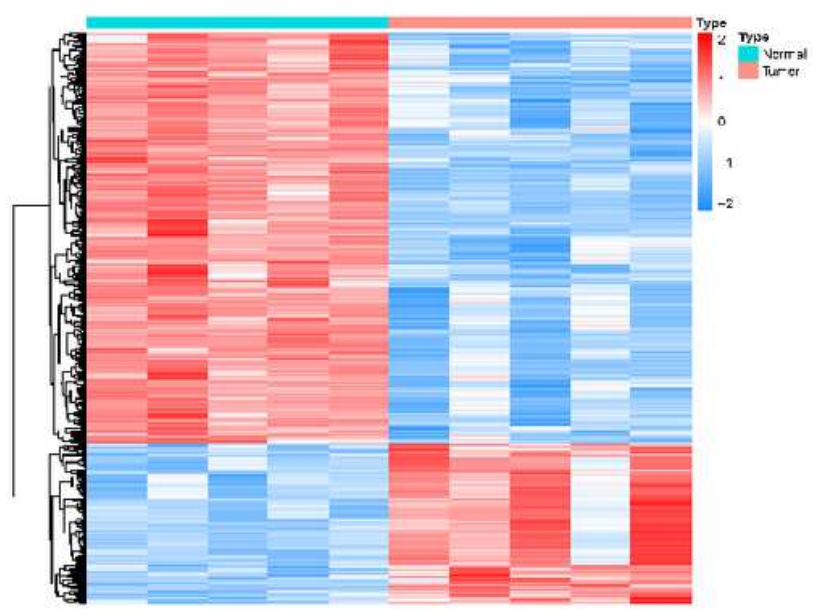

C

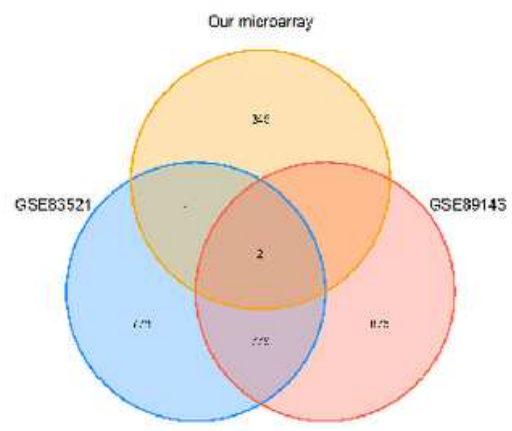

f

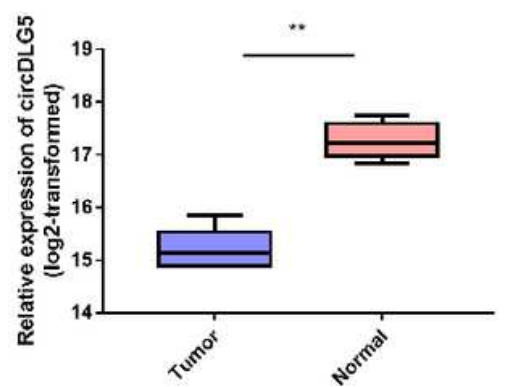

b

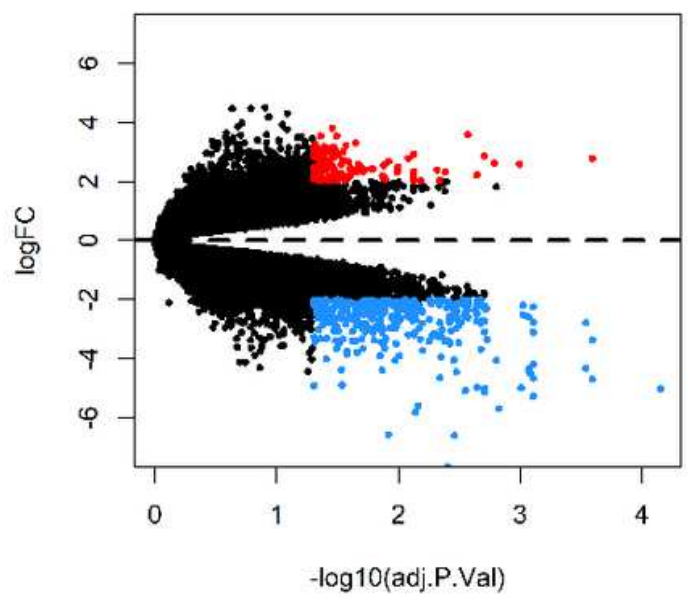

e

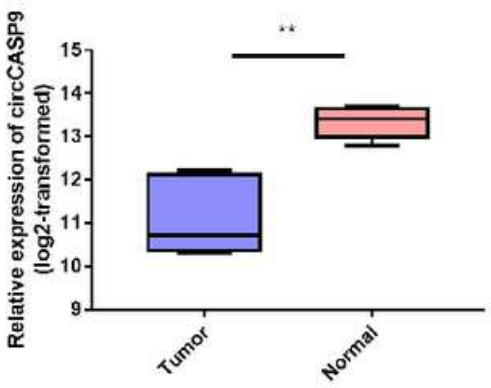

$\mathrm{h}$

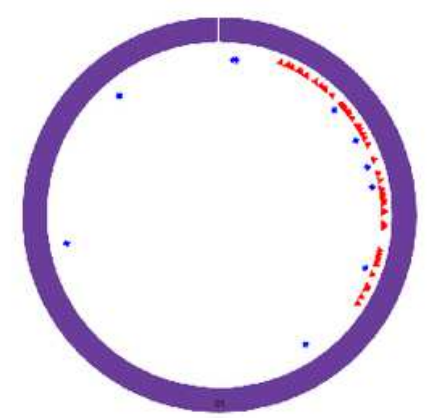

\section{Figure 1}

Identification of differentially expressed circRNAs. a Heatmap of 135 downregulated and 349 upregulated circRNAs in our microarray dataset. b Volcano map of 484 DECs in our circRNA microarray dataset. Red, blue, and black regions indicate upregulated, downregulated and not significantly different circRNAs, respectively ( $|\log \mathrm{FC}|>2$ and adj.p $<0.05)$. c, $d$ Venn diagram of downregulated and upregulated circRNAs in GSE83521, GSE89143, and our circRNA microarray datasets. Colors represent different circRNA microarray datasets. e, $f$ Expression of circCASP9 and circDLG5 in circRNA microarray datasets $\mathrm{g}, \mathrm{h}$. 
Network diagrams of circCASP9 and circDLG5 from CSCD. Red, blue, and green areas show miRNA binding sites, RNA conjunct proteins, and open reading frames (ORF), respectively.

a
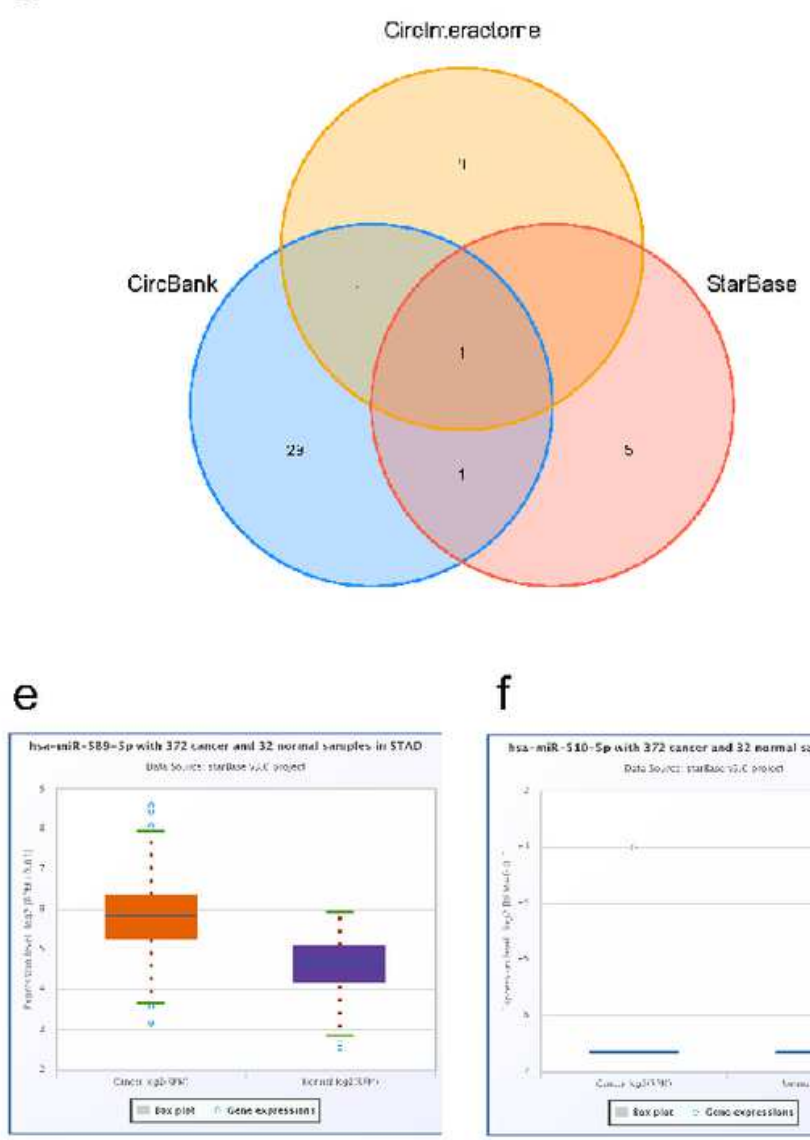

b

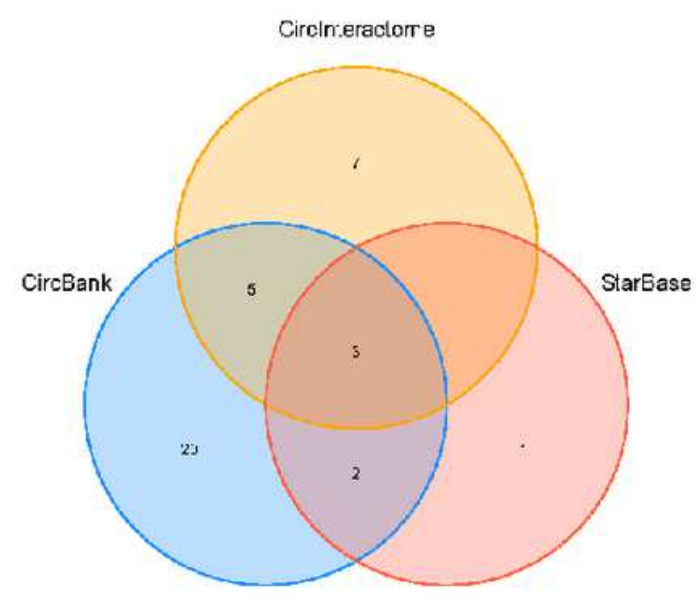

g

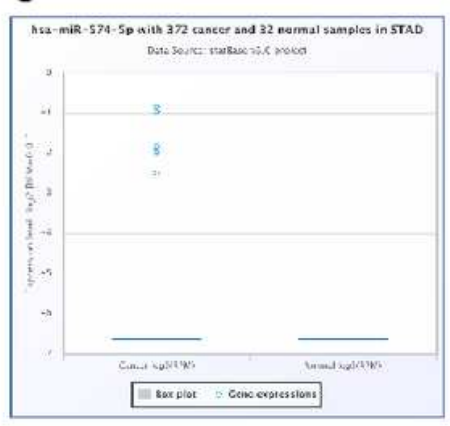

$\mathrm{h}$

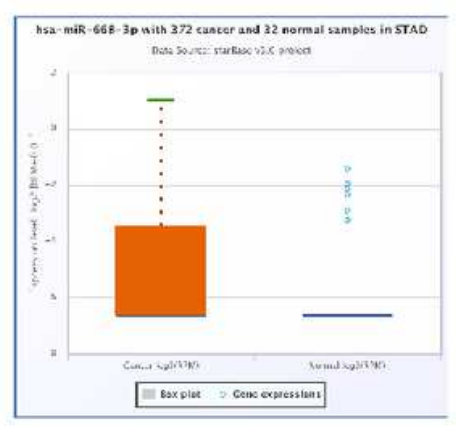

\section{Figure 2}

Identification of differentially expressed miRNAs. a, b Venn diagram of predicted miRNAs in StarBase, circBank, and Circlnteractome. e-h Expression of miRNA-589-5P, miRNA-510-5P, miRNA-574-5P miRNA668-3P in StarBase. 
a

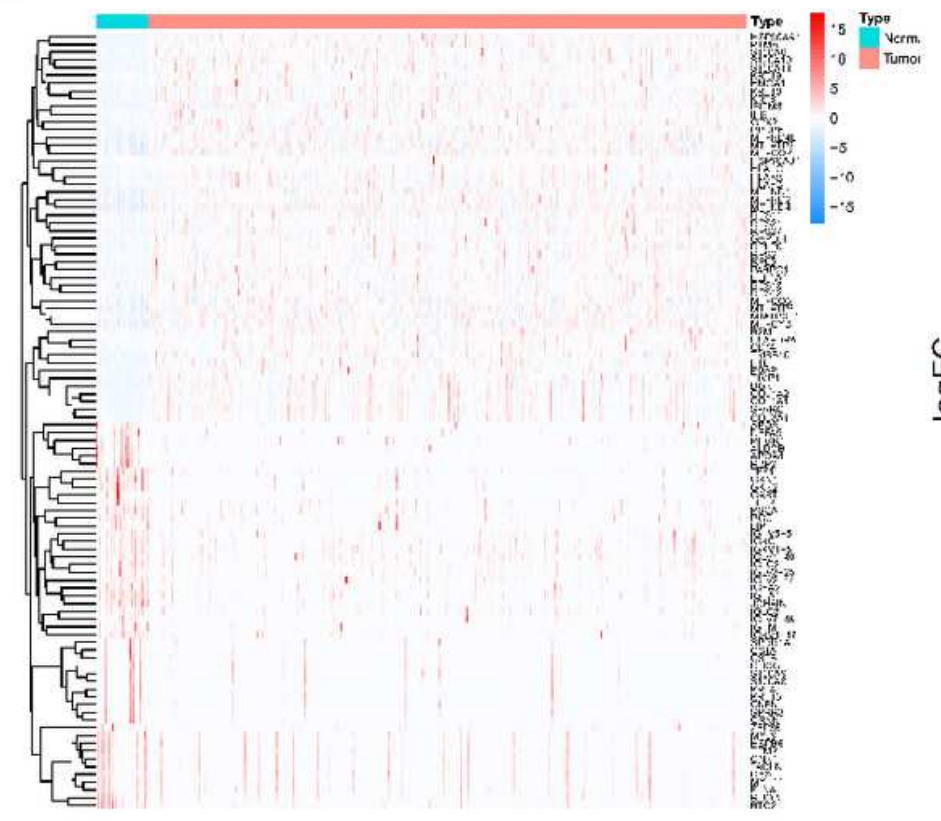

C

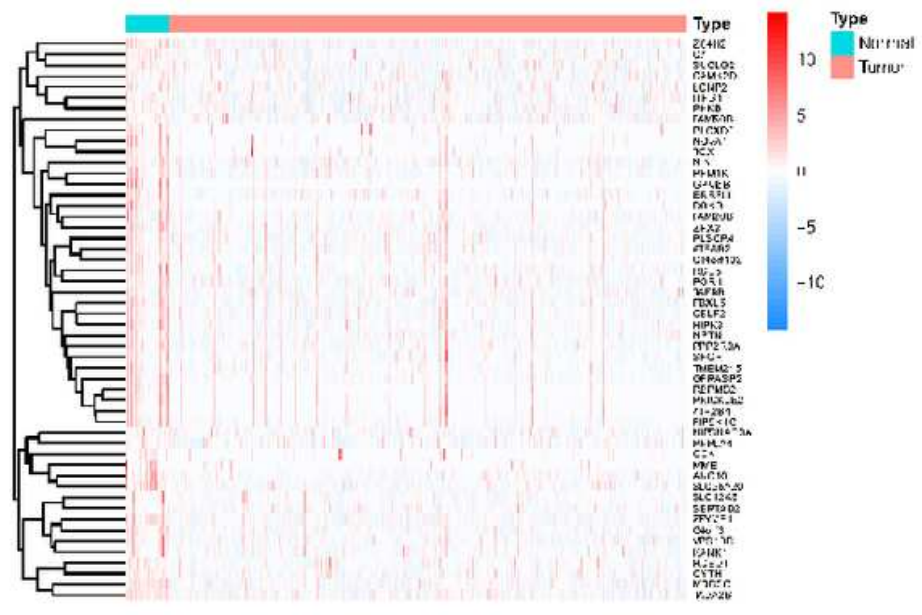

b

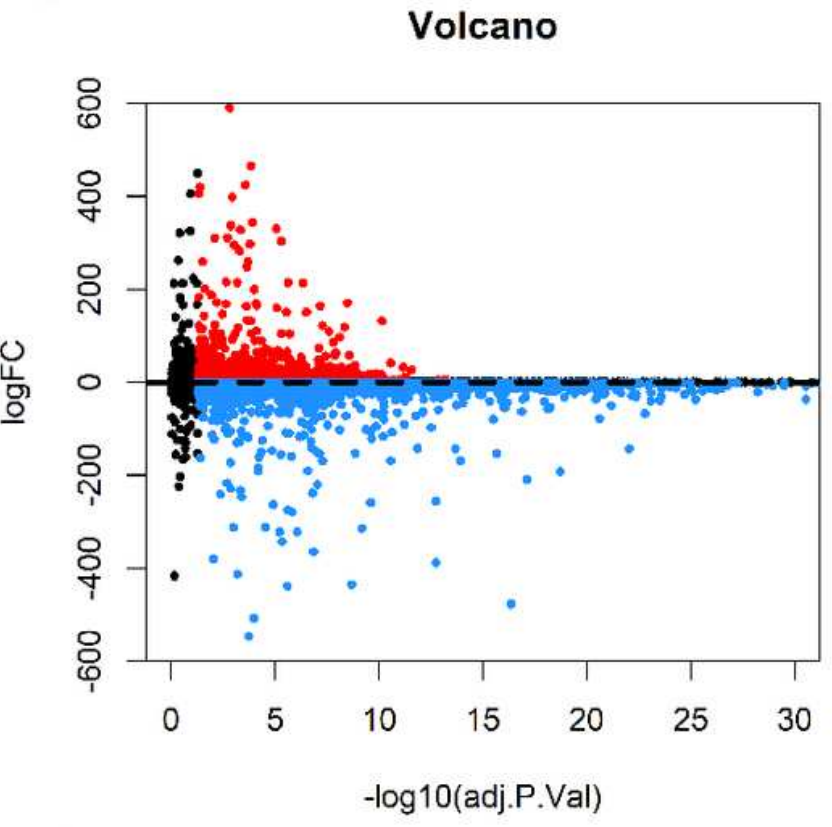

d

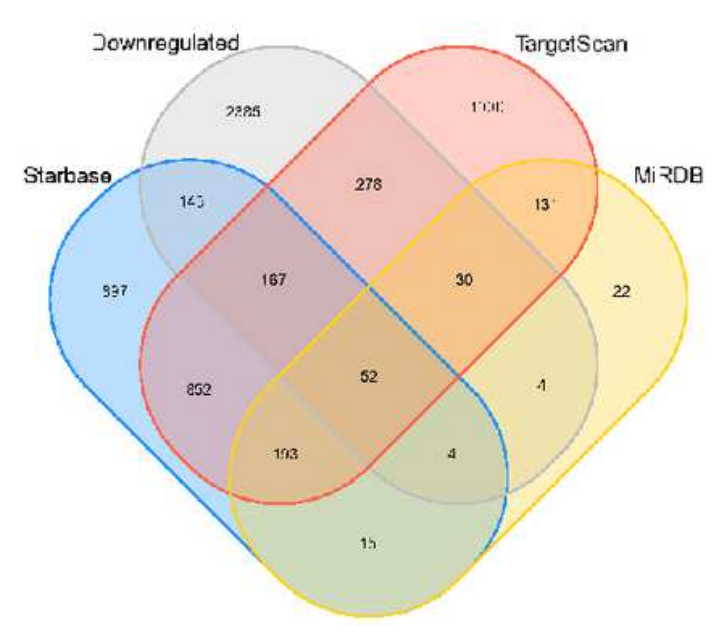

Figure 3

Identification of TMs. a, b Heatmap for top 50 DEMs, and volcano map for 3,069 downregulated and

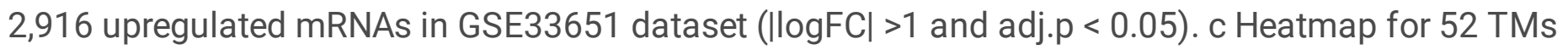
obtained from overlap of predicted mRNAs from three databases and DEMs. D Venn diagram of 52 TMs of miRNA-589-5p. 


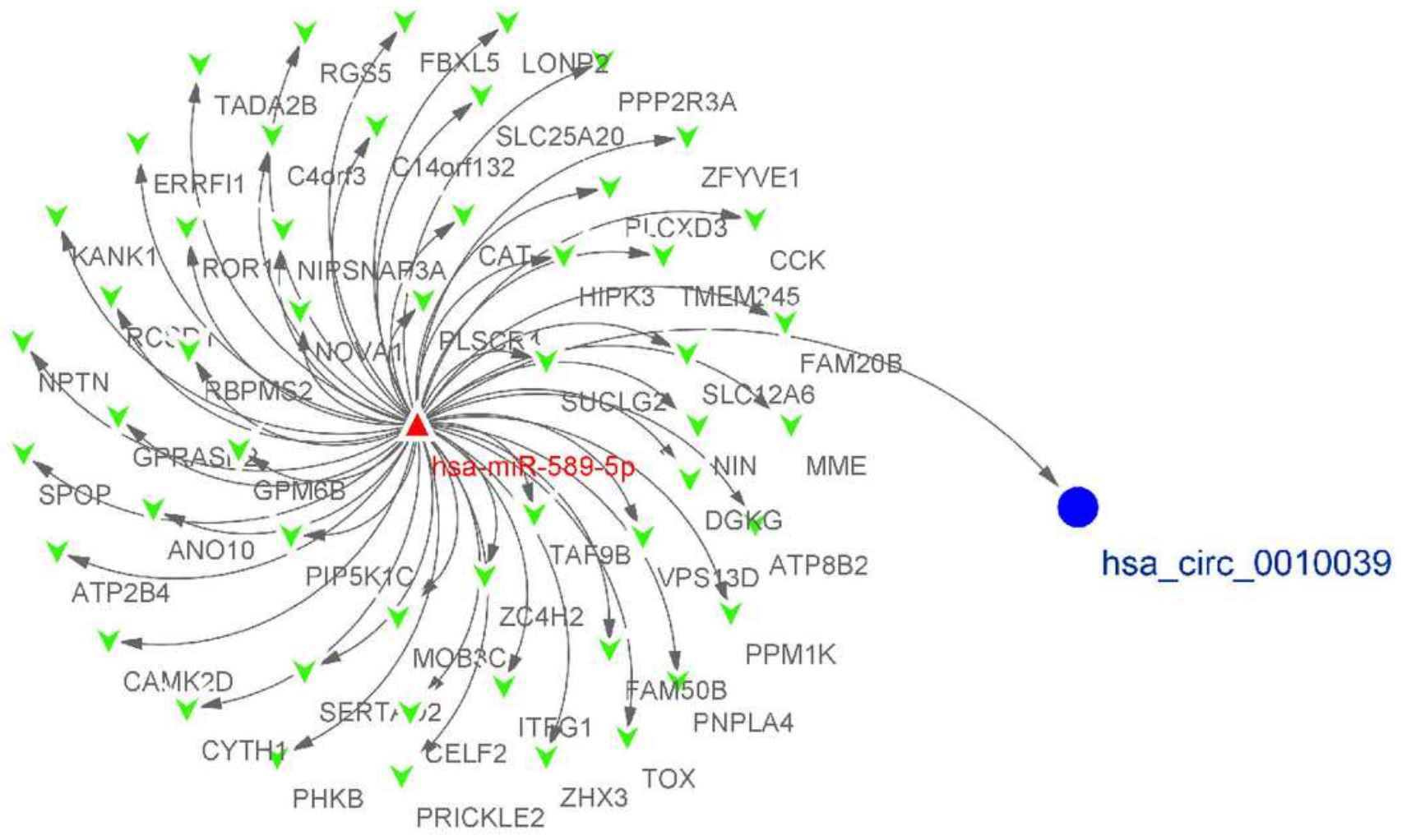

Figure 4

Circular RNA-miRNA-mRNA regulatory network established with circCASP9, miR-589-5p, and 52 mRNAs using Cytoscape. 
a

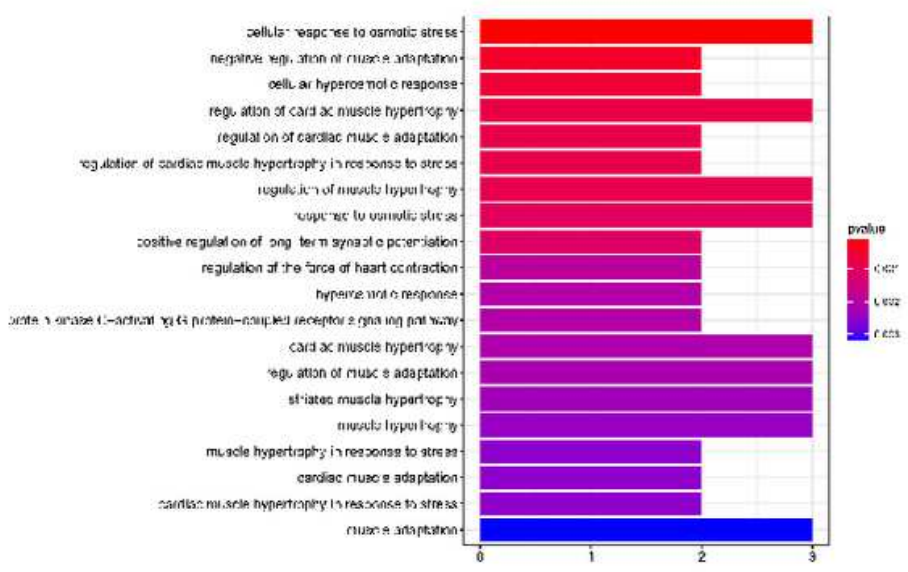

C

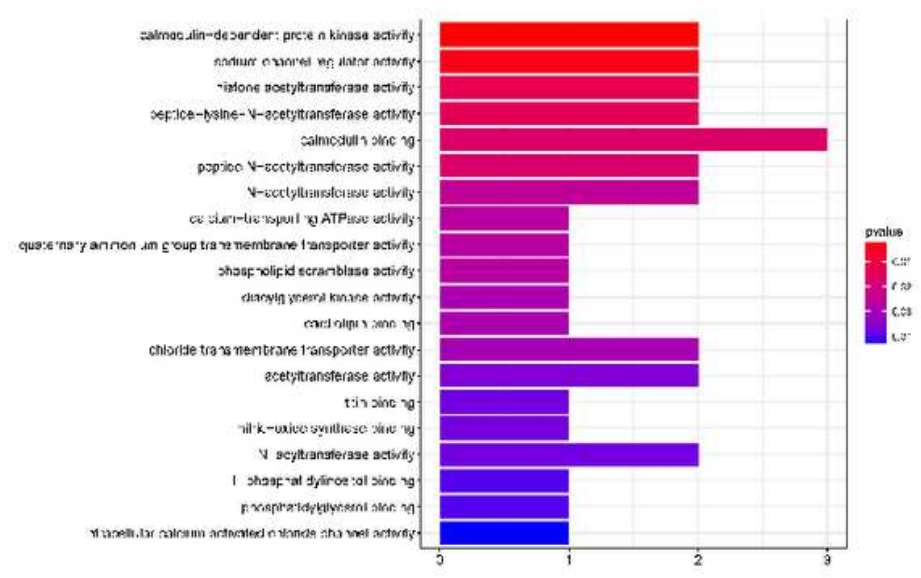

b

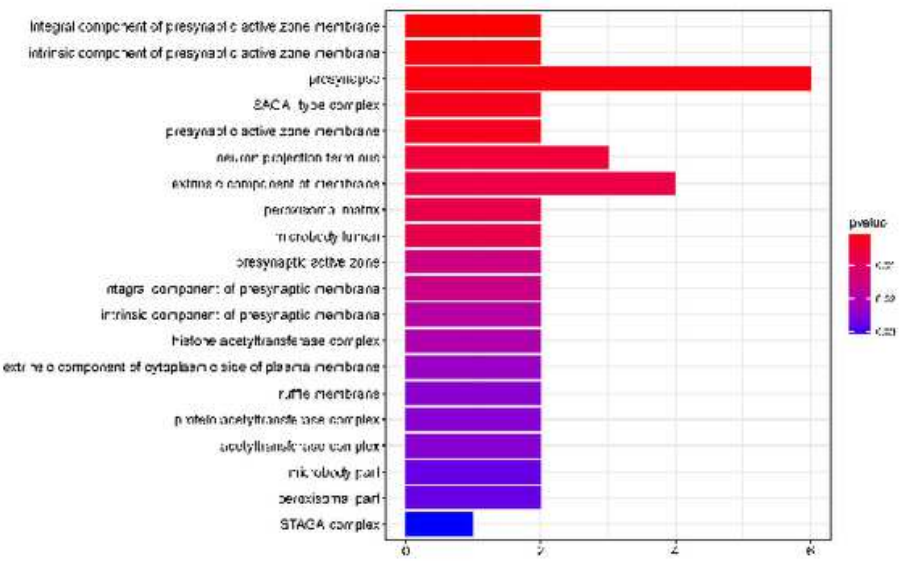

d

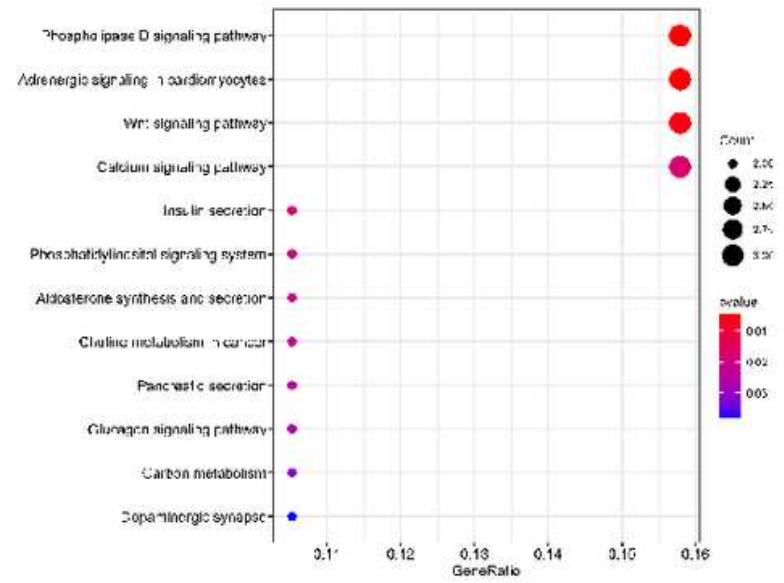

Figure 5

Gene ontology and KEGG analyses of TMs. Top 20 significantly enriched GO terms for TMs under a BP, b CC, and c MF categories. d KEGG pathways involving TMs $(p<0.05$ and $q<0.05)$. 


\section{a}

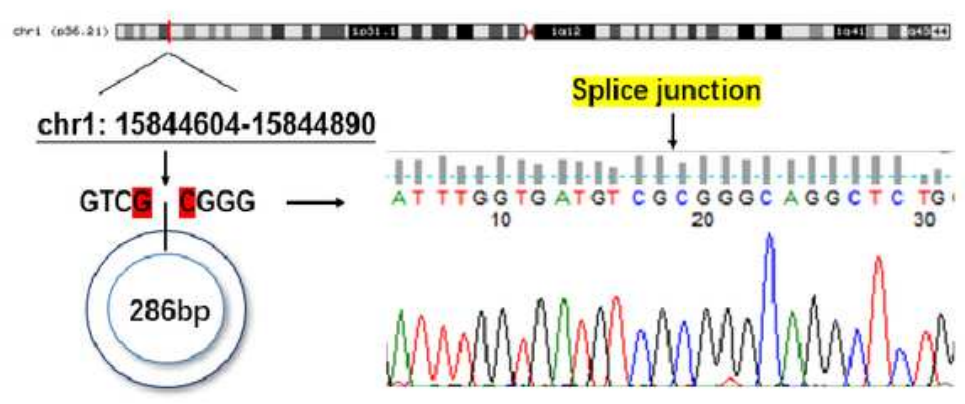

C

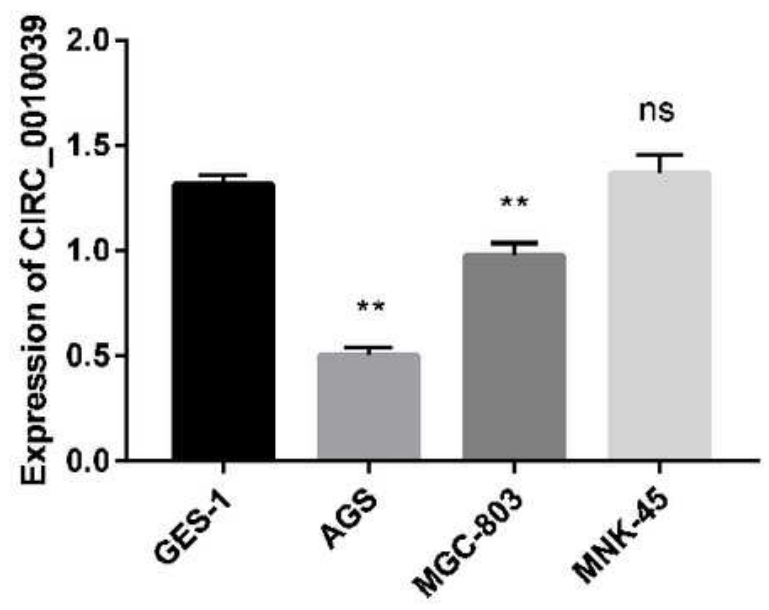

e

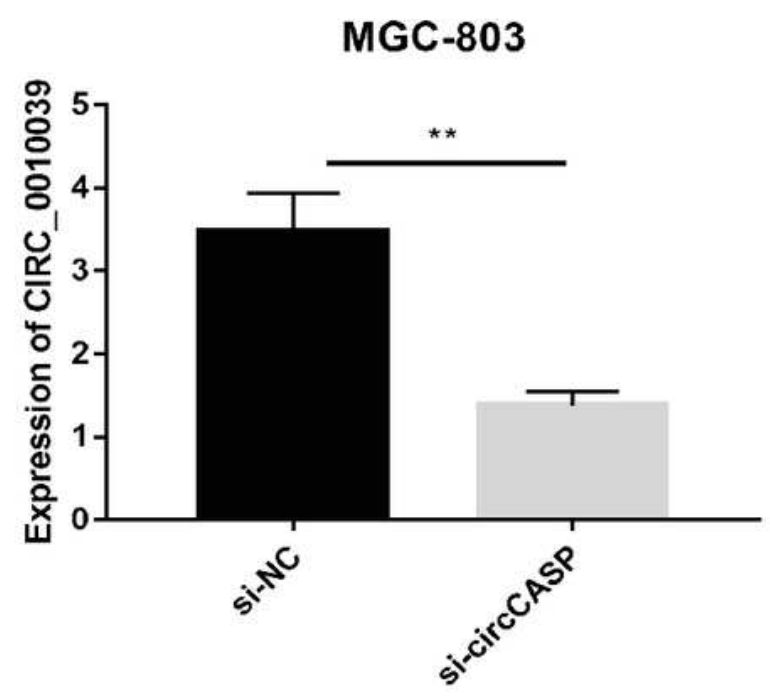

b

CASP9

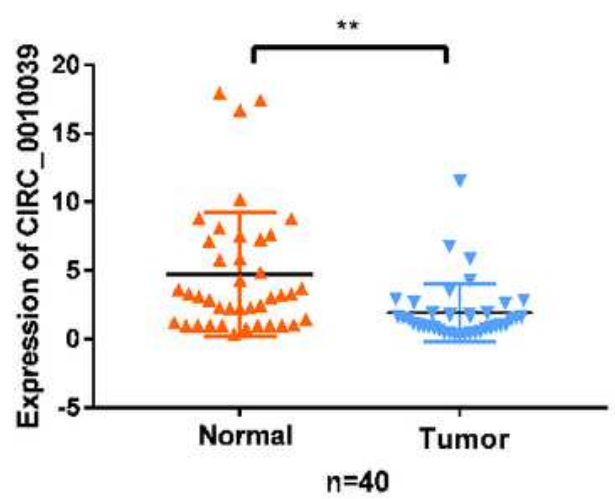

d

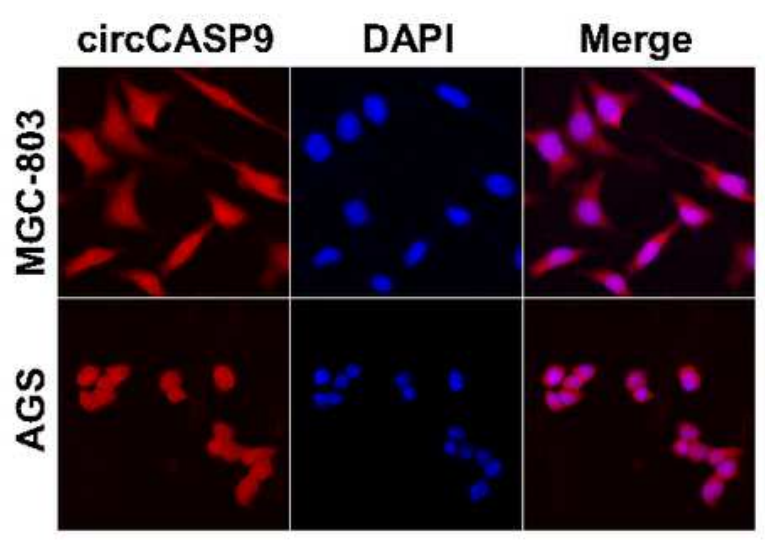

f

\section{AGS}

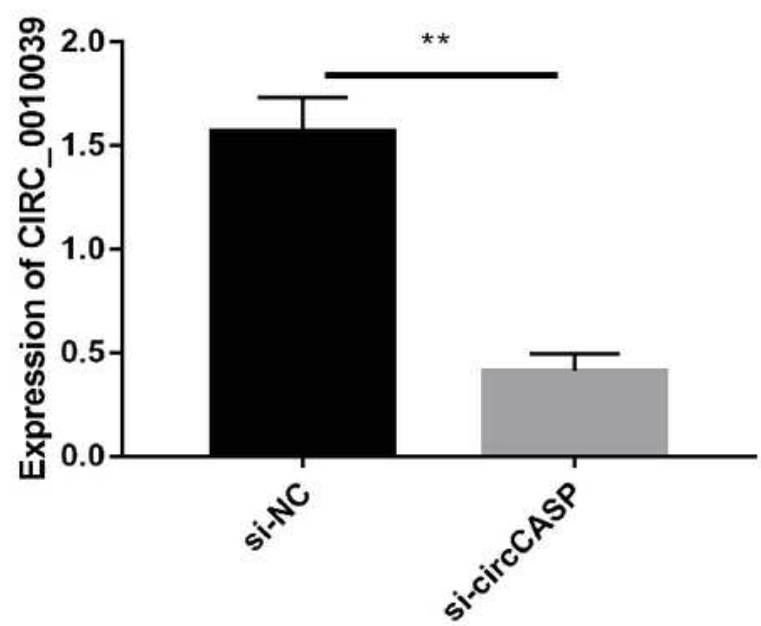

Figure 6

Characterization and expression of circCASP9. a Head-to-tail circular connection of circCASP9 verified by Sanger sequencing. b Expression of circCASP9 in b 40 paired GC and paracancerous tissues, and c AGS, MGC-803, MNK45, and GES-1 cells. d Cellular localization of circCASP9 in AGS and MGC-803 cells. e, $f$ Silencing efficacy of si-circCASP9 in AGS and MGC-803 cells. ${ }^{*} p<0.05, * \star p<0.01$. 
a

MGC-803

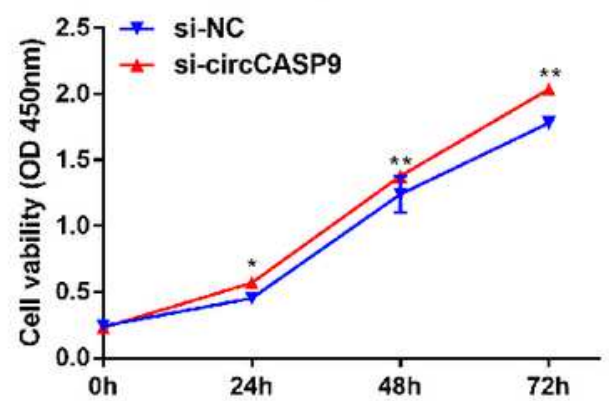

d

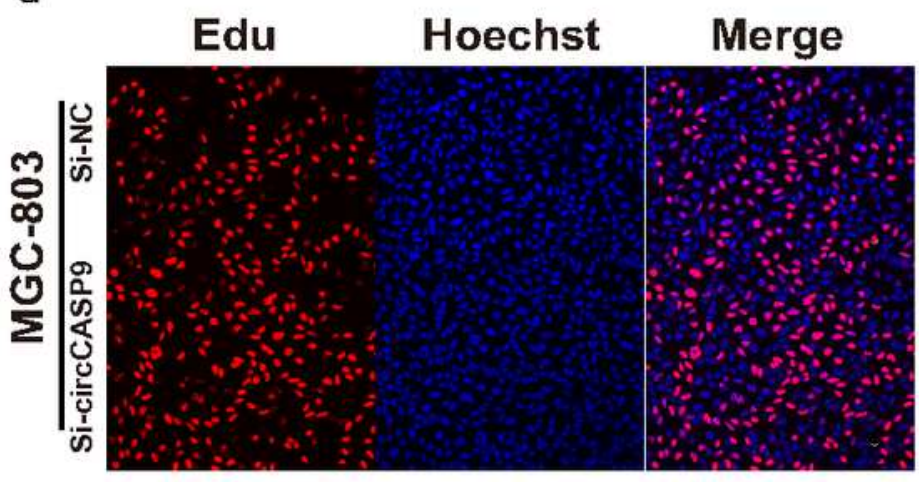

f

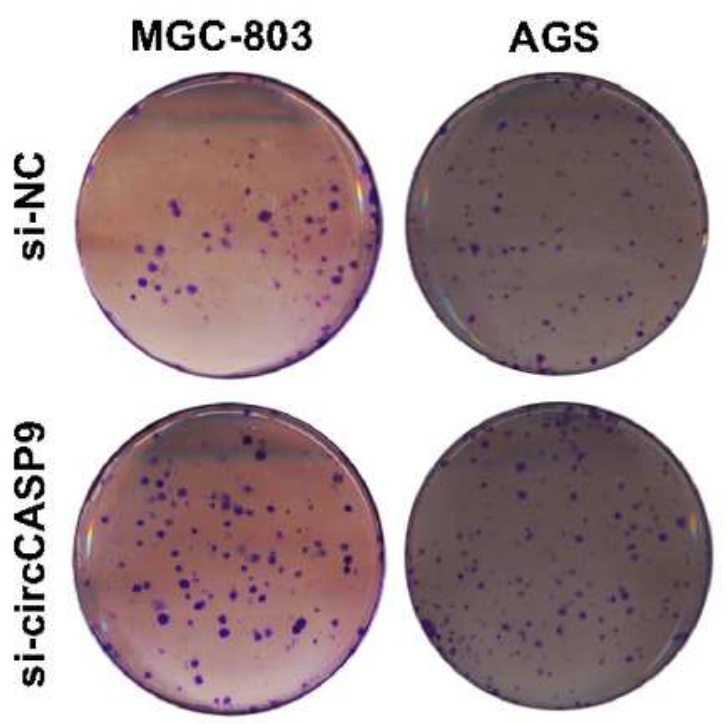

AGS

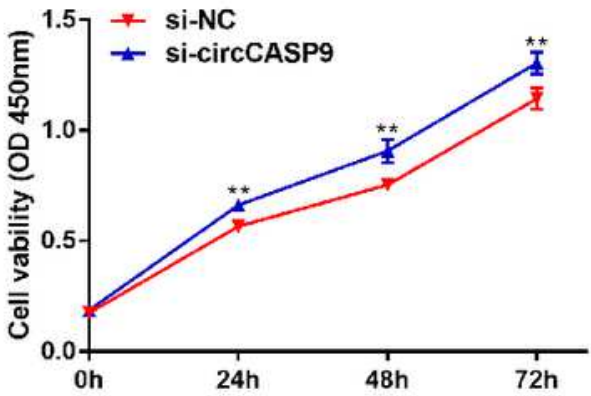

e
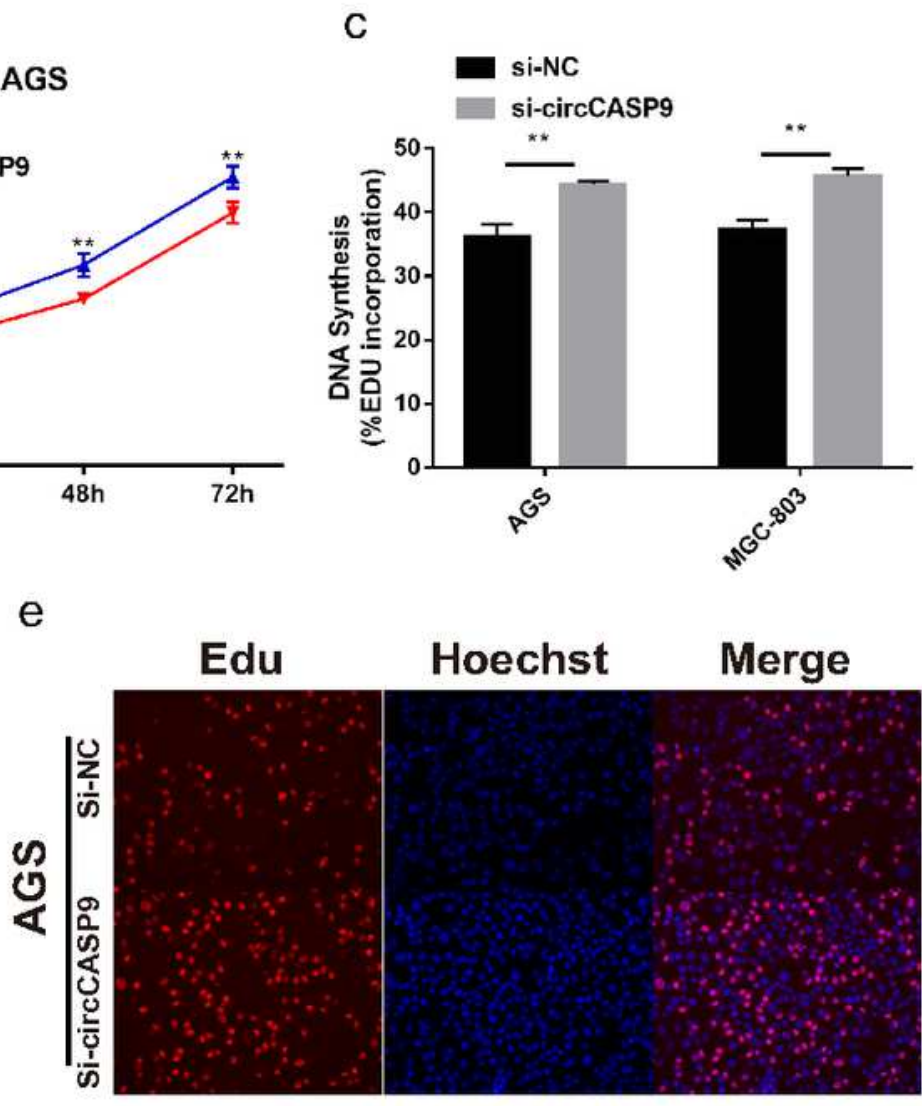

g

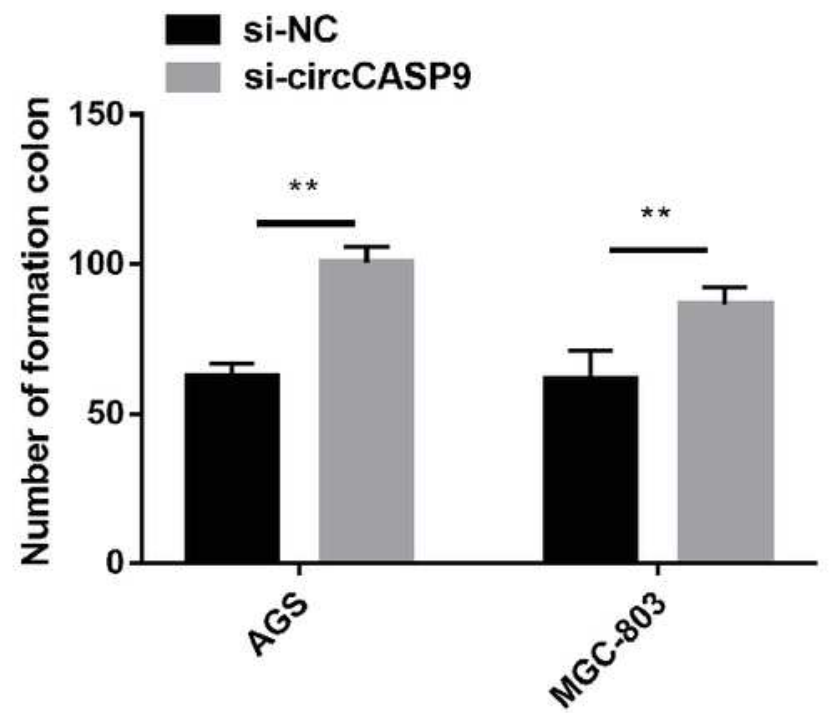

Figure 7

Circular CASP9 suppresses cell proliferation, colony formation, and DNA synthesis. a, b CCK-8 findings of the AGS and MGC-803 cells transfected with si-NC and si-circCASP9. c, d EDU assays of the AGS and MGC-803 cells transfected with si-NC or si-circCASP9. $\mathrm{f}, \mathrm{g}$ Colony formation assays of the AGS and MGC803 cells transfected with si-NC or si-circCASP9. ${ }^{*} p<0.05$, ${ }^{\star *} p<0.01$. 
a

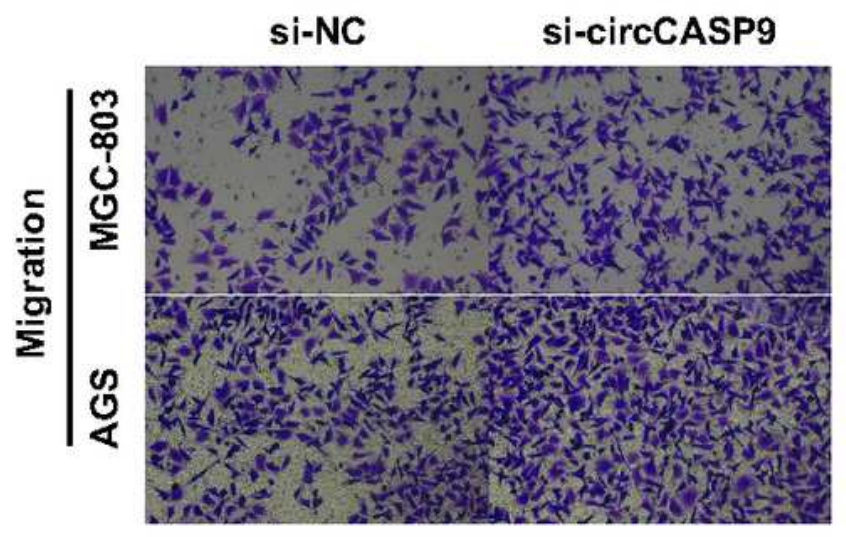

C

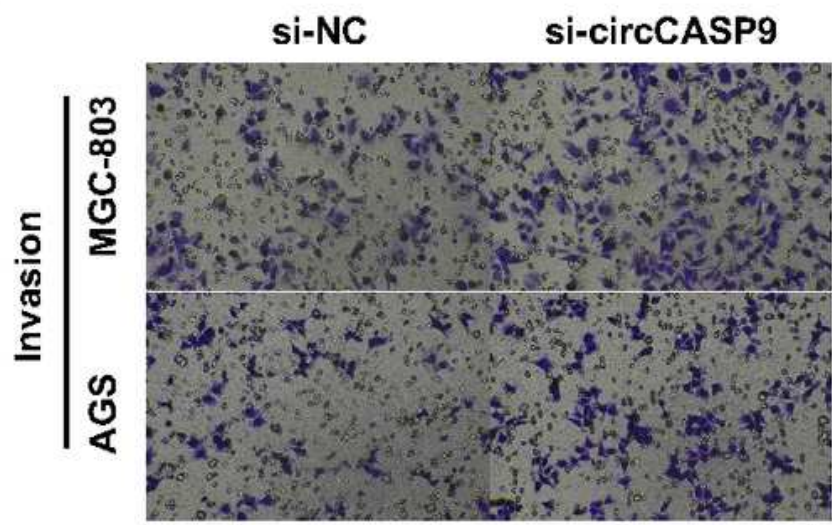

e

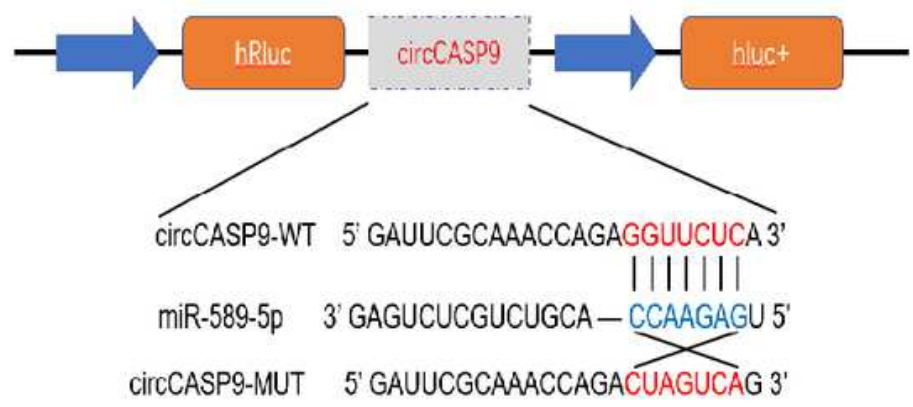

b
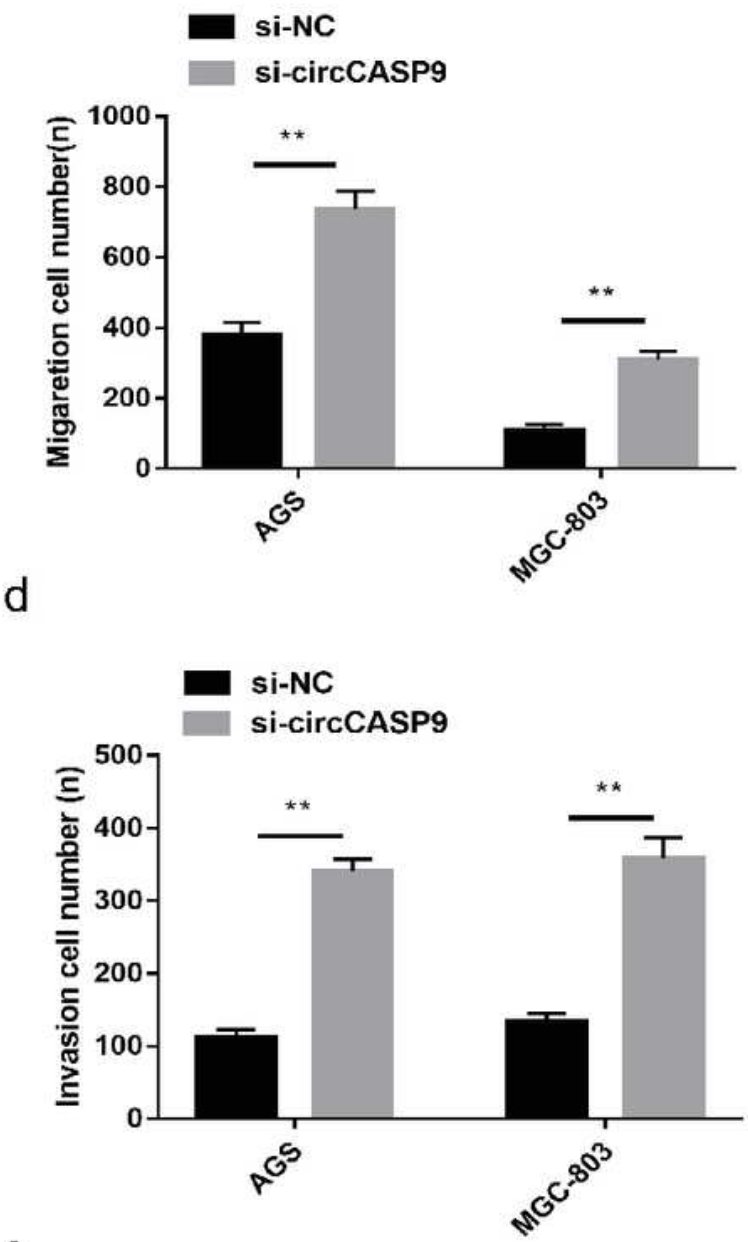

f

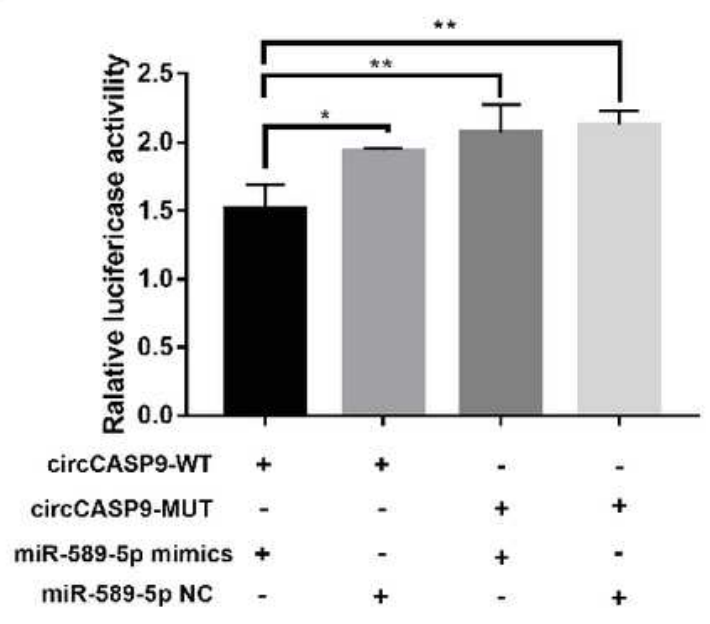

\section{Figure 8}

Cell migration and invasion were suppressed by circCASP9 and circCASP9 acted as a sponge of miR-589$5 p$ in GC cells. a Migration and b invasion of the AGS and MGC-803 cells transfected with si-NC or sicircCASP9. c Schematic representation of the potential binding sites of miR-589-5p with circCASP9. $d$ Luciferase activity of WT or Mut circCASP9 after the transfection with miR-589-5p mimic or miR-NC in MGC-803 cells. ${ }^{*} p<0.05,{ }^{\star \star} p<0.01$. 